\title{
Impact Improvement of Tape Carbon Fiber Composite Modified by Submicron Glass Fiber
}

\author{
Nhan T.T. Nguyen ${ }^{1 *}$, Naoto Miyakita ${ }^{1}$, Obunai Kiyotaka $^{1} \&$ Okubo Kazuya $^{1}$. \\ ${ }^{1}$ Department of Mechanical and Systems Engineering, Graduate school/Faculty of Science and Engineering, \\ Doshisha University, Kyotanabe, Kyoto, Japan \\ Correspondence: Nhan Thi Thanh Nguyen, Department of Mechanical and Systems Engineering, Graduate \\ school/Faculty of Science and Engineering, Doshisha University, Kyotanabe, Kyoto, Japan. Tel: 81-774-656-421. \\ E-mail: cyjb2501@mail4.doshisha.ac.jp
}

Received: September 4, 2019

doi:10.5539/jmsr.v8n4p21
Accepted: September 26, 2019 Online Published: September 30, 2019

URL: https://doi.org/10.5539/jmsr.v8n4p21

\begin{abstract}
It is well known that thermoplastic composite is vulnerable to impact fracture. Submicron glass fiber (sGF) was used to modify the matrix of chopped tape carbon fiber reinforced polypropylene composite. The impact resistance improved $20 \%$ and $7.4 \%$ coressponding to the dimeter sGF of 0.28 and $0.69 \mu \mathrm{m}$ used in modified-composite. To shed light upon the mechanism of this improvement, the internal damage statement of post-impact specimens was observed by the CT scanner. The results pointed out that the increase of the impact resistance was due to the enlargement of delamination area under impact load. The micro droplet test and end notch flexure test suggest that the decrease of Mode II fracture toughness in modified-composite comes from narrowing the difference between the interfacial shear strength (IFSS) and the bending strength of matrix thanks to significant improving of IFSS with the addition of sGF while the flexural strength remains the unchanged. Consequently, the failure mode changed from debonding fiber/matrix in unmodified composite into brittle matrix failure in modified composite, resulting in the decrease of the Mode II interlaminar fracture toughness and the enlargement of delamination area. The stress transfer test also indicates that the modified composites is prone to the brittle matrix failure.
\end{abstract}

Keywords: impact resistance, chopped tape carbon fiber (CF), submicron glass fiber (GF), polypropylene (PP) composite, modify/modifier, Mode II fracture toughness, delamination, debonding, brittle matrix failure.

\section{Introduction}

Compared to thermoset composites, thermoplastic composites offer the potential for short processing time, reparability by melting, less brittle, more tougher and higher damage tolerance (Campbell, Sih, \& Akca, 2003). Polypropylene is the most consumed polymer globally since having lowest density among commodity plastics, good resistance with fatigue and chemicals, versatile processing (Maier, Maddah, \& Huang, 2004). Therefore, carbon fiber propylene composite has been widely used in many applications such as automotive, construction, and consumer products (Kim, Gabr, \& Koncar, 2018). However, it is well-known that thermoplastic composite is vulnerable to impact fracture. Because of having the complex constituent, failure in composite under impact event is highly susceptible to crack initiation and damage propagation along the inter-laminar interfaces. The structure may fail in a wide variety of modes with the most dominant failure are the complex combinations of energy absorbing mechanisms such as (a) delamination caused by mode II shear, (b) matrix cracking resulted by transverse shear, and (c) translaminar fracture caused by fibre rupture and kinking. Among them, delamination is popular failure mode under low velocity impact (Boria, Jogur, \& Xiao-Ju, 2017). In one other statement, Walker. L et al stated that a composite structure can address the impact via two significant mechanisms: absorb energy through the creation of damaged areas and via the elastic non-failure response (Walker et al., 2002). Researchers also pointed out that there is a linear relationship between absorbed energy and delamination area (Peijs, Siow, \& Hong, 1990). Therefore, it was promoted efficient ways to improve impact resistance of composite by weakening the interlaminar bond strength or interlaminar fracture toughness to control delaminated extension (Pegoretti et al., 2008). Other mentioned methods are toughening matrix by using modifiers of thermoplastic, liquid rubber, polymer blend, hyper-branched polymer (Jang et al., 2003) and Z-pinch technique (Ye at al., 2009). In this investigation, submicron glass fiber was used to improve the impact resistance of chopped carbon fiber thermoplastic (CCT) composite. The mechanism of this improvement also was lightened by varieties of mechanical characterized tests. 


\section{Experiments}

\subsection{Materials}

Carbon fiber bundle (T700SC-12K-50C, Toray Industries, Inc. Japan) with 4.9 GPa of tensile strength and 230 $\mathrm{GPa}$ of tensile modulus was used as reinforcement. Polypropylene (PP) pellets (J708UG) were supplied by Prime Polymer Co., Ltd and kneaded with maleic acid modified polypropylene pellets (MAPP - Sanyo Kasei Co., Ltd). The modifier was submicron glass fiber (Nipon Muki Co., Ltd) and used at two different nominal diameters of 0.28 and $0.69 \mu \mathrm{m}$. PP non-woven fabric was employed as the film to wrap sGF for modifying matrix (6640-1A, Shinwa Co., Ltd).

\subsection{Preparation of PP/MAPP and modified PP/MAPP pellets}

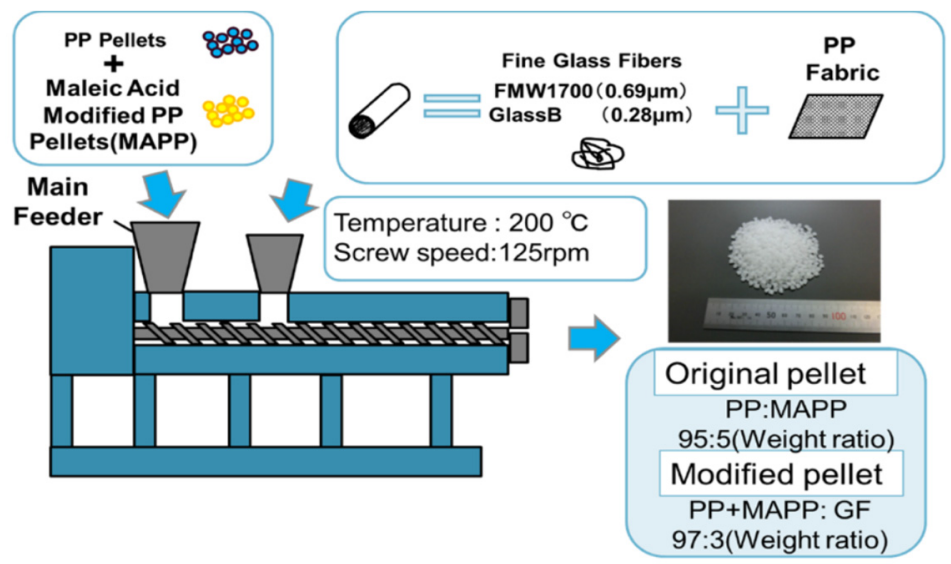

Figure 1. The schema of preparing pellets of PP/MAPP and modified PP/MAPP

To formulate pellets of PP/MAPP, PP and MAPP were put into the main-feeder of the extruder in the weight ratio of 95:5, respectively. As mentioned in the former section, the sGF was wrapped in the non-woven fabric PP and put into the sub-feeder. The weight fraction of sGF in the mixture of modified PP/MAPP was $3.0 \mathrm{wt} \%$. The processes were carried out in the twin screw kneading extruder at the speed of $125 \mathrm{rpm}, 200{ }^{\circ} \mathrm{C}$, screw diameter of $25 \mathrm{~mm}$ and the ratio of L/D was 60 . Hereinafter the type of samples was named as the original and the modified for the PP/MAPP and modified PP/MAPP, respectively.

\subsection{Fabrication of PP Sheets}

PP/MAPP and modified PP/MAPP pellets were dried at $80^{\circ} \mathrm{C}$ for 20 hours then molded at $200{ }^{\circ} \mathrm{C}$ in an extruder to form films in $50 \mu \mathrm{m}$ thick and $200-250 \mathrm{~mm}$ wide. These films were cut into smaller films in $30 \mathrm{~mm}$ width after that.

\subsection{Preparation of Uni-direction Tape}

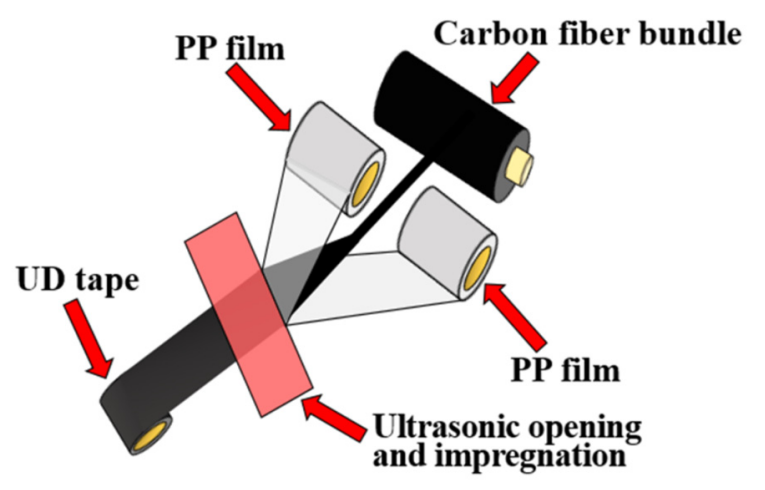

Figure 2. Process of preparing uni-direction (UD) tape of carbon fiber reinforced PP 
Figure 2 shows the process of making uni-direction tape from coils of PP film and carbon fiber. Under the orientation force, PP films as well as carbon fiber were straight stretched and fiber was interleaved between two films to form the UD tape. The ultrasonic device was put at the beginning contact-point of fiber and resin film to facilitate spreading of fiber and impregnating of resin over carbon fiber. The volume fraction of fiber in tape was $30 \%$.

\subsection{Fabrication of chopped carbon fiber tape (CTT) composite}
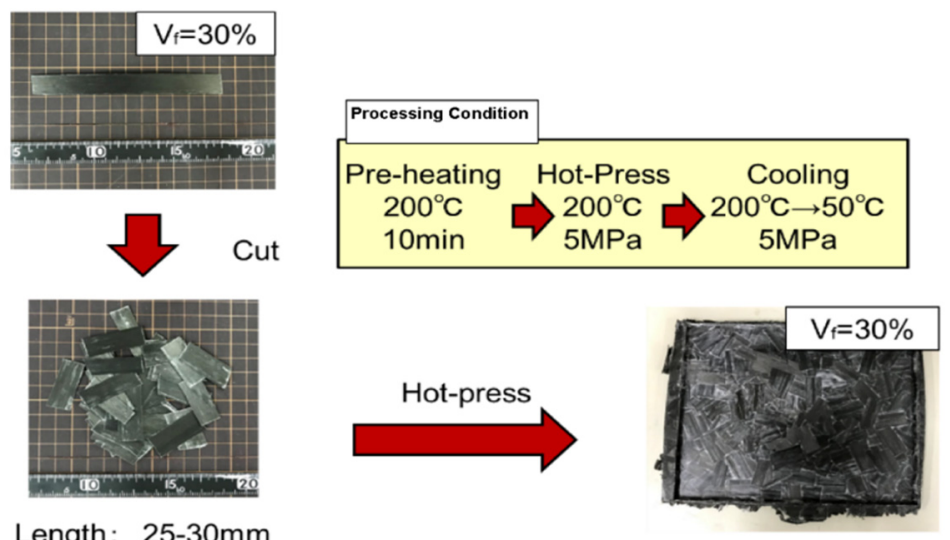

Length: $25-30 \mathrm{~mm}$ Width $15-20 \mathrm{~mm}$

Thickness:4mm

Figure 3. Preparation process CTT composite from UD tape.

The UD tape was cut into 25-30 mm long like strip shape then dispersed onto a mold at random. The mold was heated to $200{ }^{\circ} \mathrm{C}$, kept for 10 minutes on the heat press machine followed by applying a pressure of $5 \mathrm{MPa}$ in 5 minutes and naturally cooled down then to room temperature to form plate in dimensions of $250 \times 200 \times 4 \mathrm{~mm}^{3}$ while the pressure was kept constant.

\subsection{Izod Impact Resistance Test}

The specimens for Izod impact test were cut from the CTT plate in dimensions of $80 \times 10 \times 4 \mathrm{~mm}^{3}$ by the diamond cutter. One flatwise impacting of $5.5 \mathrm{~J}$ energy was applied on the edge of specimen. The test followed the standard of JIS K7110. At least 10 specimens were used for each condition.

\subsection{Evaluation of Damage State After Impact}

To evaluate the state of internal damage after impact test, a small piece with the length and width of $20 \mathrm{~mm}$ and $10 \mathrm{~mm}$, respectively was cut from the post-impacted specimen containing fracture area. The fracture surface was observed by the X-ray CT scanner and the delamination area was calculated by using the CT image analysis software.

\subsection{Microdroplet Test}

Figure 4 shows the geometry of specimen for micro-droplet test. Specimens were subjected to access the interfacial shear strength between matrix and single fiber. Testing speed was $0.12 \mathrm{~mm} / \mathrm{min}$. Test data were obtained with at least 20 samples under each condition.

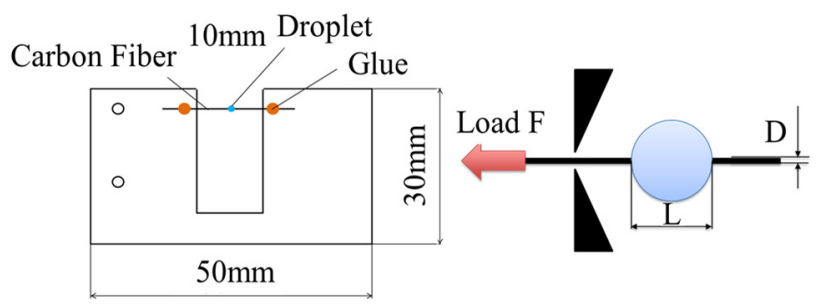

Figure 4. Specimen for micro-droplet test. 
2.9 End notch Flexure (ENF) Test

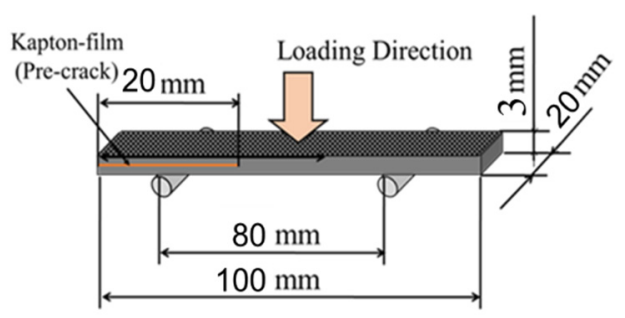

Figure 5 The geometry of sample for ENF test.

The geometry of ENF sample is shown in the Figure 5. Whereas the Izod impact test, the ENF test was conducted on the uni-directional specimens which was cut from the UD-fiber composite plate. To prepare UD-plate, 30 sheets of UD tape were hot-pressed in the same condition with that of CTT composite along with a film of teflon in 20 $\mathrm{mm}$ long was inserted between the $15^{\text {th }}$ layer and $16^{\text {th }}$ layer to achieve sample in dimensions of $100 \times 20 \times 3 \mathrm{~mm}^{3}$. The test was performed under the three point bending load at speed of $0.5 \mathrm{~mm} /$ minute. The Mode II interlaminar fracture toughness $\mathrm{G}_{\text {IIC }}$ is calculated by the following equations:

$$
\begin{gathered}
a_{1}=\left[\frac{C_{1}}{C_{0}} a_{0}^{3}+\frac{2}{3}\left(\frac{C_{1}}{C_{0}}-1\right) L^{3}\right]^{\frac{1}{3}} \\
G_{I C}=\frac{9 a_{1}^{2} P_{C}^{2} C_{1}}{2 B\left(2 L^{3}+3 a_{1}^{3}\right)}
\end{gathered}
$$

Wherein:

$\mathrm{a}_{0}$ : initial crack length $(\mathrm{mm})$

$\mathrm{P}_{\mathrm{C}}$ : Initial limit load $(\mathrm{N})$

$\mathrm{C}_{0}$ : Load point compliance of initial elastic part $(\mathrm{mm} / \mathrm{N})$

$\mathrm{C}_{1}$ : Load point compliance at initial limit load $(\mathrm{mm} / \mathrm{N})$

$\mathrm{a}_{1}$ : Estimated crack growth length at initial limit load (mm)

L: Distance between load point and support point $(\mathrm{mm})$

B: Specimen width $(\mathrm{mm})$

\subsection{Stress Transfer Test on the Model Sample}

To observe the stress transfer around the carbon fiber ends, a model composite was prepared by sandwiching a carbon fiber bundle in $20 \mathrm{~mm}$ long between two PP films followed by melting resin and consolidating sample in the vacuum furnace. The geometry of sample is shown in the Figure 6. Pre-cracks with crack length approximate $2 \mathrm{~mm}$ was prepared by the diamond cutter at both sides then natural cracks introduced by slicing razor at the precrack root before testing. The sample surface was painted by white and black paints to create a speckle pattern for digital image correlation (DIC) analysis. The tensile load was applied to the model specimen at a constant cross head speed of $1 \mathrm{~mm} / \mathrm{min}$.

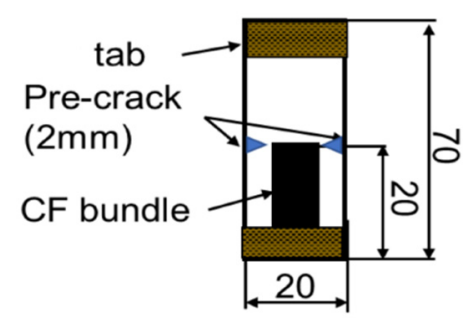

Figure 6. The model composite sample for stress transfer test 


\section{Results and Discussions}

\subsection{Izod Impact Resistance}

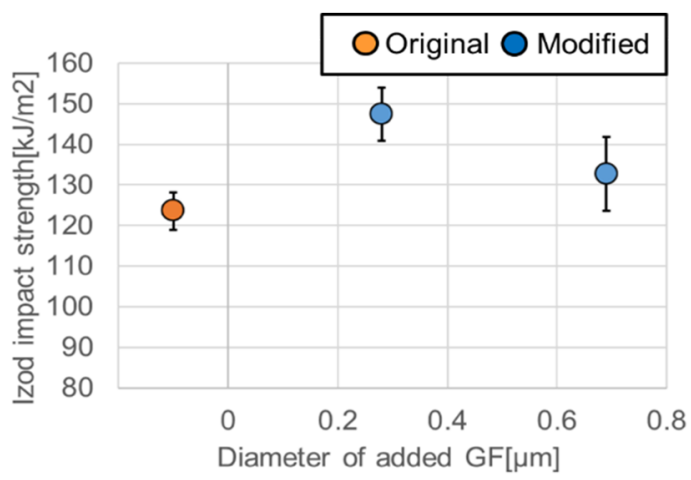

Figure 7. The Izod impact resistance of the original and the modified of CTT composite with respect to the diameter of sGF

As can be seen from the Figure 7, the Izod impact resistance was improved with the addition of sGF into matrix in composite. The increase was inversely propotional to the increase of the diameter sGF. There was the increment of $20 \%$ and $7.4 \%$ coressponding to the dimeter sGF of 0.28 and $0.69 \mu \mathrm{m}$ used. This indicates that the efficiency of improving impact resistance in chopped carbon fiber tape reinforced PP is dependent on the size of sGF modifier.

\subsection{Evaluation of Damage State After Impact}
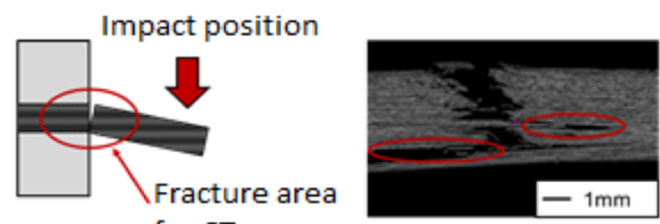

for CT scan

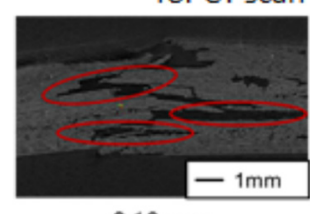

$0.18 \mu \mathrm{m}$

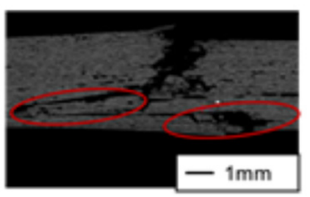

$0.69 \mu \mathrm{m}$

Figure 8 The CT images of fracture surfaces after impact test

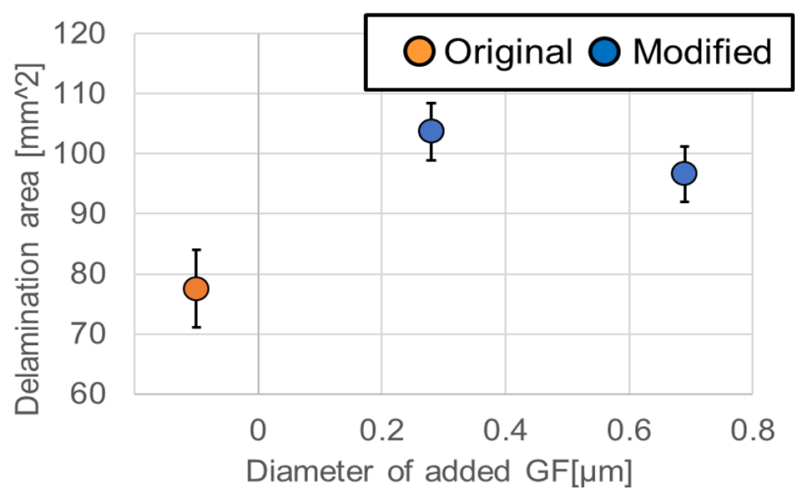

Figure 9. The delamination area after impact test of the original and the modified of CTT composite with respect to the diameter of sGF 
From the Figure 8, it can be seen that the addition of sGF changed the failure mode of composite samples. The crack propagated through the surface of specimen in the straight direction in the original but in the zig-zag form in the modified composite. In the other words, the delamination seemed more serious in the case of the modified CTT. The calculation of damage area also showed the same tendency with the impact resistance of composites, the delamination area increased $33.7 \%$ and $24.6 \%$ with the composite modified by sGF in diameter of 0.28 and $0.69 \mu \mathrm{m}$, respectively compared to that of the unmodified composite (see Figure 9). This suggests that adding sGF into PP matrix enlarges the delamination area, resulting in the improvement of impact resistance in modified composites.

\subsection{The Interfacial Shear Strength (IFSS) between Fiber and Matrix}

There was the significant increment of IFSS between fiber and matrix when the resin was modified with sGF. The degree of improvement of IFSS was related to the diameter of glass fiber as same as that of impact resistance. With sGF in the diameter of $0.28 \mu \mathrm{m}$, the improvement was $83 \%$ while that of $0.69 \mu \mathrm{m}$, IFSS increased $62 \%$ compared to the interface strength of un-modified resin. Normally, the increase of interface adhesion leads to improvement of impact performance. However, an optimum level of interface bonding is required for best impact performance since high amount of IFSS makes composite brittle (Jogur et al., 2018). In this research, the improvement of impact resistance was tendentiously similar to the increment of IFSS indicated that the SGF was used in the suitable ratio in PP matrix to enlarge impact performance of CTT.

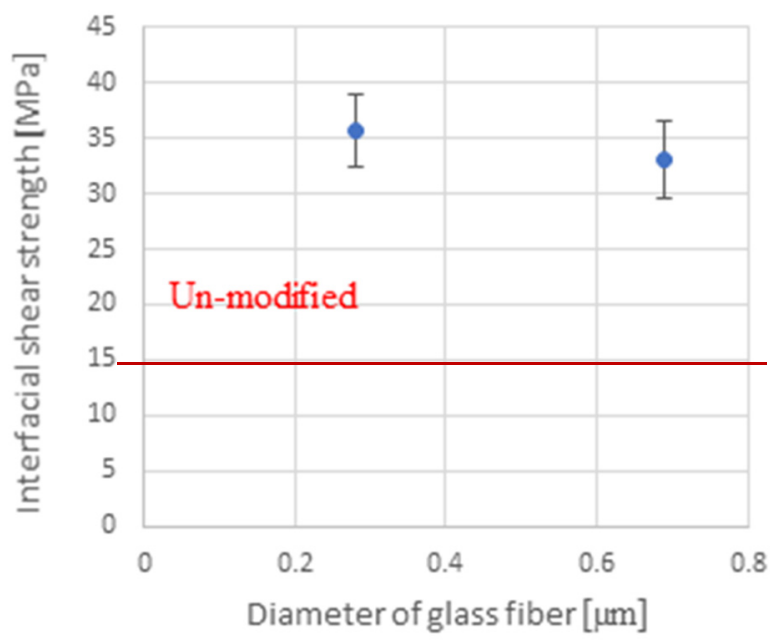

Figure 10. The IFSS between single fiber and matrix with respect to different diameter of sGF.

\subsection{ENF Test}

For the sake of understanding the enlargement of delamination area under the impact load, the end notch flexure test was conducted. The interlaminar Mode II fracture toughness of uni-direction fiber composite decreased when the PP matrix was modified with glass fiber (Figure 11). However, as pointed out in the Figure 12, the images of SEM observation showed the reversed manner: more resin left on the fiber surface in the case of modified UD fiber composites. In the other words, there was the weak adhesion between fiber and matrix in un-modified resin composite. As mentioned by Madhukar et al. in the research about graphite/epoxy composite, the stronger adhesion between fiber and matrix the higher interlaminar fracture toughness. However, when the IFSS reaches the optimum level, the brittle failure of matrix can be observed, causes the decrease of Mode II fracture toughness (Madhukar et al., 1992). The decline of Mode II fracture toughness can be assumed the difference between IFFS and bending strength of matrix is narrowed with the addition of sGF. In the case of un-modified matrix, the value of IFSS was approximately $19 \mathrm{MPa}$ while that of bending strength of PP was $40 \mathrm{MPa}$. However, the IFSS was suddenly increased up to $35.6 \mathrm{MPa}$ and $33.2 \mathrm{MPa}$ for matrixes added with the glass fiber in the diameter of 0.28 and 0.69 $\mu \mathrm{m}$, respectively while the bending strengths remained the unchanged (see Figure 13). Therefore, the dominant fracture mode in unmodified UD-fiber composite was debonding between fiber and matrix. Whereas, the interfacial fracture turned into brittle matrix fracture in the case of modified composite. This explains the increase of delamination area or the decrease of Mode II fracture toughness occurred in UD-fiber composite modified by submicron glass fiber. 


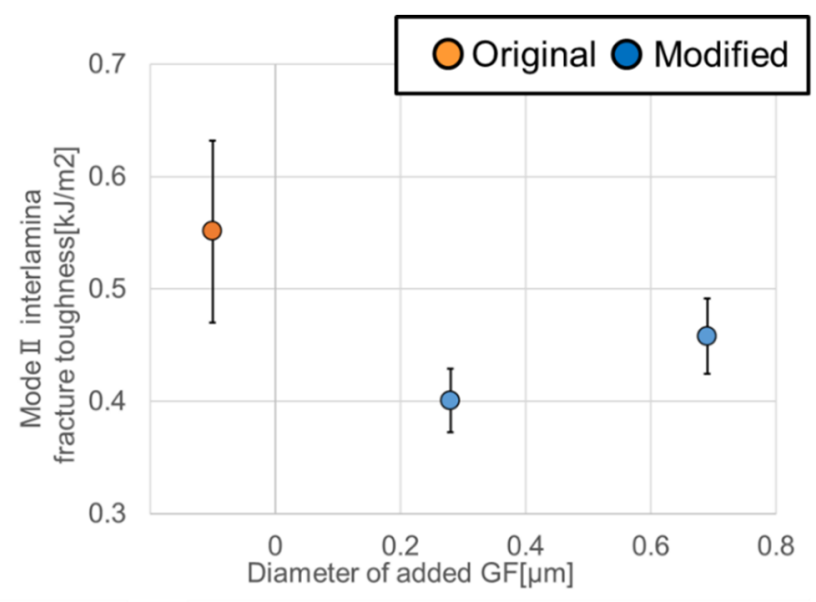

Figure 11. Mode II interlaminar fracture toughness of UD-fiber composite with respect to diameter of sGF

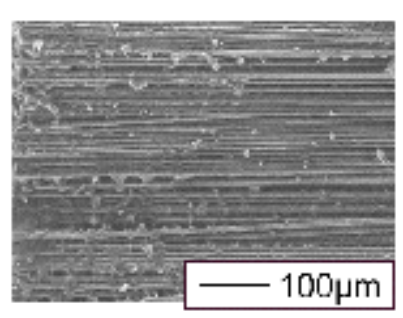

Un-modified

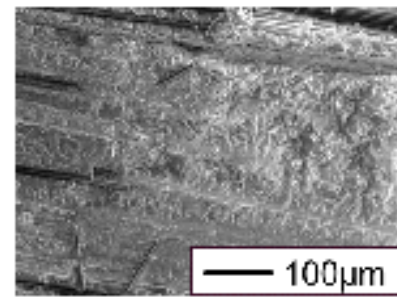

$0.28 \mu \mathrm{m}$

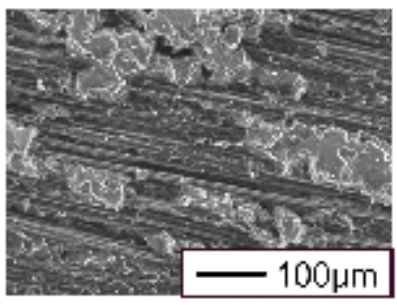

$0.69 \mu \mathrm{m}$

Figure 12. The SEM images of fracture surfaces after ENF test

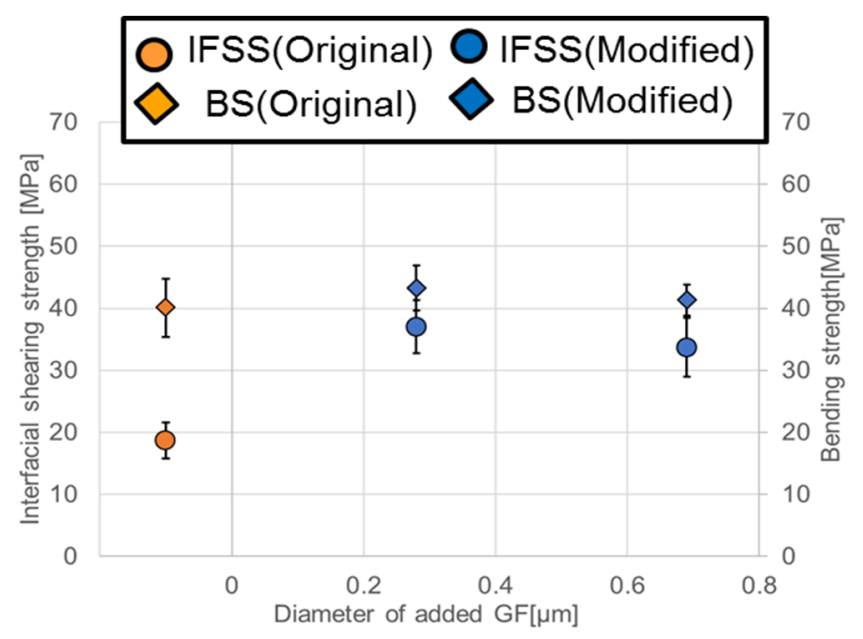

Figure 13. The correlation between IFSS and the bending strength of polypropylene

\subsection{The Stress Transfer in the Model Specimens}

As can see in the Figure 14, there was the distinct difference in images achieved from the DIC analysis between the un-modified and modified specimen at the stage of just before failure. In the modified specimen, the stress mainly concentrated at pre-cracks while extending to the vicinity of fiber bundle end in the case of un-modified specimen. Images of specimens after failure were pointed out in the right side of Figure 14. Two cracks on the both sides of the original model also connected to form the final failure but there was the deflection of crack, the 
failure occurred at the weak parts of resin for the modified model. The test of model specimens shows that the modified composites are prone to the brittle failure. These results are also good agreement with that of ENF test.

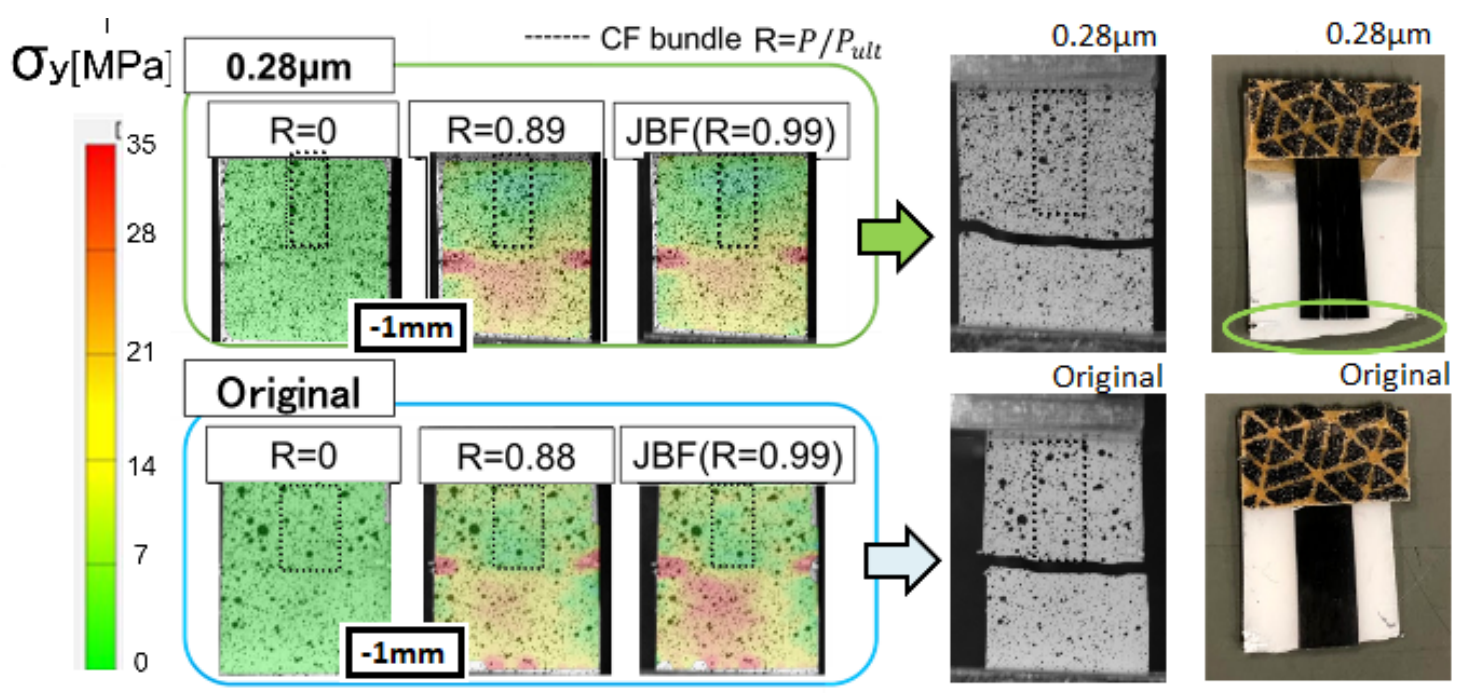

Figure 14. The strain distribution test in the model specimens of original composite and the sGF modified composite

\section{Conclusions}

Submicron glass fiber was added into matrix in chopped CF tape reinforced PP composite. Compared to unmodified composite, the impact resistance improved $20 \%$ and $7.4 \%$ when sGF in 0.28 and $0.69 \mu \mathrm{m}$ diameter used in composite, respectively. The mechanism of this increase was brightened by varieties of mechanical tests. The results are summarized as follows:

1. The delamination area in composites under impact load rose with the addition of sGF and reached the highest in the case of composite modified by sGF in $0.28 \mu \mathrm{m}$ diameter.

2. The micro droplet test and the ENF test pointed out that the difference between the interfacial shear strength fiber/matrix and the bending strength was narrowed by the significant improvement of the IFSS value while the flexural strength of resin remained the unchanged with the addition of glass fiber. Therefore, the dominant failure mode in composite changed from debonding fiber/resin for the un-modified to brittle matrix failure for the modified. These results explain the decrease of the Mode II fracture toughness in modified UD fiber composite, leads to the enlargement of delamination area and improvement of impact resistance in modified CTT composite.

3. In the specimen model made from the single fiber bundle and PP matrix, two notches connected to form the final failure in un-modified model. However, there was the deflection of crack, the failure occurred at the weak parts of resin for the modified model. This proves the modified composite is prone to the brittle matrix failure.

\section{Acknowledgments}

The authors gratefully acknowledge Nippon Muki Co., Ltd for the financial and material supports provided to this research and master student Mr. Ueda Taiki for supporting experiments.

\section{References}

Akca, E., \& Gursel, A. (2015). A Review on the Matrix Toughness of Thermoplastic Materials. Periodicals of Engineering and Natural Sciences (PEN), 3(2), 15-27. doi: 10.21533/pen.v3i2.52

Boria, S., Scattina, A., \& Belingardi, G. (2017). Impact behavior of a fully thermoplastic composite. Composite Structures, 167, 63-75. doi: 10.1016/j.compstruct.2017.01.083

Campbell, F. (2003). Manufacturing processes for advanced composites (pp. 357-397). Burlington: Elsevier.

Gabr, M., Okumura, W., Ueda, H., Kuriyama, W., Uzawa, K., \& Kimpara, I. (2015). Mechanical and thermal properties of carbon fiber/polypropylene composite filled with nano-clay. Composites Part B: Engineering, 69, 94-100. doi: 10.1016/j.compositesb.2014.09.033 
Hong, S., \& Liu, D. (1989). On the relationship between impact energy and delamination area. Experimental Mechanics, 29(2), 115-120. doi: 10.1007/bf02321362

Huang, C., Lou, C., Liu, C., Huang, C., Song, X., \& Lin, J. (2015). Polypropylene/Graphene and Polypropylene/Carbon Fiber Conductive Composites: Mechanical, Crystallization and Electromagnetic Properties. Applied Sciences, 5(4), 1196-1210. doi: 10.3390/app5041196

Jang, S., Adachi, T., \& Yamaji, A. (2003). Impact Damage Resistance of CFRP Laminate with Epoxy-Resin Surface Layer. Journal of The Society of Materials Science, Japan, 52(6Appendix), 138-142. doi: 10.2472/jsms.52.6appendix_138

Jogur, G., Khan, A., Das, A., Mahajan, P., \& Alagirusamy, R. (2018). Impact Properties of Thermoplastic Composites. Textile Progress, 50(3), 109-183. doi: 10.1080/00405167.2018.1563369

Kim, B., Deka, B., Bae, I., Choi, D., Son, D., \& Park, Y. (2018). Unidirectional spread-tow carbon fiber/polypropylene composites reinforced with mechanically aligned multi-walled carbon nanotubes and exfoliated graphite nanoplatelets. Polymer Composites, 39(S2), E1251-E1261. doi: 10.1002/pc.24835

Koncar, V. (2018). Smart textiles for in situ monitoring of composites (1st ed., pp. 154-215). Woodhead Publishing.

Maddah, A. (2016). Polypropylene as a Promising Plastic: A Review. American Journal of Polymer Science, 6(1), 1-11. doi: 10.5923/j.ajps.20160601.01

Madhukar, M., \& Drzal, L. (1992). Fiber-Matrix Adhesion and Its Effect on Composite Mechanical Properties: IV. Mode I and Mode II Fracture Toughness of Graphite/Epoxy Composites. Journal of Composite Materials, 26(7), 936-968. doi: 10.1177/002199839202600701

Maier, C., \& Calafut, T. (2001). Polypropylene (1st ed., pp. 87-144). Norwich, N.Y: Plastics Design Library.

Pegoretti, A., Cristelli, I., \& Migliaresi, C. (2008). Experimental optimization of the impact energy absorption of epoxy-carbon laminates through controlled delamination. Composites Science and Technology, 68(13), 2653-2662. doi: 10.1016/j.compscitech.2008.04.036

Peijs, A., Venderbosch, R., \& Lemstra, P. (1990). Hybrid composites based on polyethylene and carbon fibres Part 3: Impact resistant structural composites through damage management. Composites, 21(6), 522-530. doi: 10.1016/0010-4361(90)90425-v

Sih, G., Carpinteri, A., Pantelakis, S., Kermanidis, T., Spathopoulos, T., \& Baxevani, E. (1995). Advanced technology for design and fabrication of composite materials and structures (pp. 365-375). Dordrecht: Kluwer Academic Publishers.

Siow, Y., \& Shim, V. (1998). An Experimental Study of Low Velocity Impact Damage in Woven Fiber Composites. Journal of Composite Materials, 32(12), 1178-1202. doi: 10.1177/002199839803201203

Walker, L., Sohn, M., \& Hu, X. (2002). Improving impact resistance of carbon-fibre composites through interlaminar reinforcement. Composites Part A: Applied Science and Manufacturing, 33(6), 893-902. doi: $10.1016 / \mathrm{s} 1359-835 \times(02) 00010-6$

Xiao-Yu, S., Jian-Xin, T., Zheng, H., \& Xuan, G. (2018). A Study on the Failure Mechanisms of Composite Laminates Simultaneously Impacted by Two Projectiles. Advanced Composites Letters, 27(3), 96-107. doi: $10.1177 / 096369351802700302$

Ye, Y. P., Chen, H. B., Wu, J. S., Chan, C. -., \& Ye, L. (2009). Properties of advanced fiber composites with halloysite nanotube toughened epoxy matrix. Paper presented at the Seventeenth ICCM International Conferences on Composite Materials, Edinburgh, Scotland (UK). Retrieved from www.scopus.com

\section{Copyrights}

Copyright for this article is retained by the author(s), with first publication rights granted to the journal.

This is an open-access article distributed under the terms and conditions of the Creative Commons Attribution license (http://creativecommons.org/licenses/by/4.0/). 